Table 1 Data review: separating the percentage entry for two EPR systems and paper across relevant fields (Wu et al.) [1]
Table 2 Summary of our data in eye casualty record quality audit (UHCW)

\begin{tabular}{llllc}
\hline & $\begin{array}{l}\text { Medisoft }(\%) \\
n=170\end{array}$ & $\begin{array}{l}\text { Open eyes }(\%) \\
n=100\end{array}$ & $\begin{array}{l}\text { Paper }(\%) \\
n=170\end{array}$ & $\begin{array}{l}\chi^{2} p \text {-values Paper versus } \\
\text { Medisoft }(\text { Open eyes })\end{array}$ \\
\hline Intraocular pressure & 98.8 & 90 & 100 & $0.50(<0.001)^{\mathrm{a}}$ \\
Central corneal thickness & 80.6 & 85 & 85.9 & $0.19(0.84)$ \\
Gonioscopy & 62.35 & 64 & 88.8 & $<0.001(<0.001)$ \\
Fundus examination & 88.8 & 69 & 90 & $0.72(<0.001)$ \\
Past medical history & 81.17 & 58 & 92.4 & $0.002(<0.001)$ \\
Current medications & 80.58 & 47 & 93.5 & $<0.001(<0.001)$ \\
Glaucoma medications & 78.8 & 28 & 88.2 & $0.19(<0.001)$ \\
Drug allergies & 78.8 & 38 & 87.6 & $0.03(<0.001)$ \\
\hline
\end{tabular}

${ }^{a}$ Fisher exact test

\begin{tabular}{lcll}
\hline & $\begin{array}{l}2016 \\
n=100\end{array}$ & $\begin{array}{l}2017 \\
n=100\end{array}$ & $\begin{array}{l}\chi^{2} p \text {-values 2016 } \\
\text { versus 2017 Medisoft entries }\end{array}$ \\
\hline Presenting complaint & 99 & 85 & $<0.001$ \\
Past ocular history & 70 & 57 & 0.06 \\
Past medical history & 63 & 38 & $<0.001$ \\
Drug history & 27 & 22 & 0.41 \\
Allergies & 49 & 21 & $<0.001$ \\
Family history & 19 & 5 & 0.002 \\
Social history & 12 & 5 & 0.04 \\
Diagnosis & 95 & 93 & 0.55 \\
Prescription recorded & 100 & 99 & $1^{\mathrm{a}}$ \\
Outcome recorded & 98 & 96 & 0.41 \\
\hline
\end{tabular}

\title{
Response to: Comment on 'How do paper and electronic records compare for completeness? A three centre study'
}

\author{
Clara Hoi Ka Wu $\mathbb{1}^{1} \cdot$ Sheila M. H. Luk ${ }^{2} \cdot$ Richard L. Holder $^{3} \cdot$ Zena Rodrigues $^{4} \cdot$ Faisal Ahmed ${ }^{4} \cdot \operatorname{lan}$ Murdoch $\mathbb{1}^{2}$
}

Received: 9 April 2019 / Accepted: 9 April 2019 / Published online: 10 May 2019

(c) The Royal College of Ophthalmologists 2019

Clara Hoi Ka Wu

clarahoika.wu@gmail.com

Whittington Health NHS Trust, London, UK

2 Moorfields Eye Hospital NHS Foundation Trust, London, UK

3 Guy's and St Thomas' NHS Foundation Trust, London, UK

4 Imperial College Healthcare NHS Trust, London, UK
To the Editor,

We thank Qadir and Kadyan for their comments concerning our study [1]. The interesting point is that the data they share shows exactly the same findings as we report. With specific reference to our methodology:

1. We sampled the data over three sites with two different EPR systems as we believe this reduced the bias of analysis based on a specific EPR. 
2. Data collection was over period of time that allowed staff training and familiarisation of the systems as they were being introduced.

3. Our Table 1 showed the results of both EPR systems separately and combined together to allow readers to interpret the data in detail independently. For the Moorfields City Road data, we identified the samples of new patients with a stamp in the medical notes to indicate full documentation of the entry on EPR, based on the discretion of the clinicians. The fact IOP was only recorded in $90 \%$ of the Openeyes entry we speculate was due to it not being a forced choice option.

4. Both electronic and paper data entry in all three sites can be performed by clinicians, trained ophthalmic nurses and technicians. The final entries were all completed by the clinicians. For the paper entry, structured paper new patient proformas were used across three sites.

Following on from our study, one of the centres (Western Eye Hospital) has carried out incremental changes to the EPR documentation in the outpatient glaucoma service. Consent forms are currently scanned and outcome sheets are being made electronic. This programme has now been rolled out to the whole ophthalmology department making it paperless/paperlight.

Whilst we agree electronic records are the "way of the future', the findings of both our study and the findings of Qadir and Kadyan highlight the need for regular and continuous evaluation of the electronic system. This evaluation will ensure safety and reliability in the transition to a 'paperless', or at least a 'paperlight', NHS.

\section{Compliance with ethical standards}

Conflict of interest The authors declare that they have no conflict of interest.

Publisher's note: Springer Nature remains neutral with regard to jurisdictional claims in published maps and institutional affiliations.

\section{References}

1. Qadir M, Kadyan A. Comment on 'How do paper and electronic records compare for completeness? A three centre study'. Eye 2019. https://doi.org/10.1038/s41433-019-0465-4

\title{
Macular toxicity secondary to occupational exposure to gold melting
}

\author{
Hussein Almuhtaseb ${ }^{1} \cdot$ Georgios Tsokolas ${ }^{2} \cdot$ Andrew Lotery $^{3}{ }^{3}$. Assad Jalil ${ }^{4}$
}

Received: 27 April 2019 / Accepted: 2 May 2019 / Published online: 20 May 2019

(c) The Royal College of Ophthalmologists 2019

\section{Case Presentation}

A 60-year-old man was referred to the Vitreoretinal Department of the Manchester Royal Eye Hospital due to

\section{Andrew Lotery}

a.j.lotery@soton.ac.uk

1 Manchester Royal Eye Hospital, Oxford Road, Manchester M13 $9 \mathrm{WL}, \mathrm{UK}$

2 Leicester Royal Infirmary Eye Unit, Leicester Royal Infirmary, Leicester LE1 5WW, UK

3 Southampton Eye Unit, Southampton General Hospital, Tremona Road, Southampton SO16 6YD, UK

4 Manchester Royal Eye Hospital, Oxford Road, M13 9WL Manchester, UK progressive distortion in the left eye. He also complained of increasing difficulty in reading and also of the presence of dark spots in his central vision left worse than the right eye.

The patient's visual symptoms were first attributed to his left cataract but after cataract surgery, he became more aware of a central scotoma.

Of note, the patient mentioned that he worked in the jewelry industry and one of his major tasks was the melting of pure gold. He admitted that occasionally he melted gold without wearing protective goggles, although usually he wore eye protection. He also denied exposure to bright sunlight or looking at the sun for a long time, and he denied any other laser exposure.

On clinical examination, his best corrected visual acuity (BCVA) was 0.7 and 0.9 LogMAR right and left, respectively. Pupillary reflexes were normal and the rest of the 